\title{
Treatment-resistant and Multi-therapy resistant criteria for bipolar depression: A consensus definition - CORRIGENDUM
}

Diego Hidalgo-Mazzei, Michael Berk, Andrea Cipriani, Anthony J. Cleare, Arianna Di Florio, Daniel Dietch, John R. Geddes, Guy M. Goodwin, Heinz Grunze, Joseph F. Hayes, Ian Jones, Siegfried Kasper, Karine Macritchie, R. Hamish McAllister-Williams, Richard Morriss, Sam Nayrouz, Sofia Pappa, Jair C. Soares, Daniel J. Smith, Trisha Suppes, Peter Talbot, Eduard Vieta, Stuart Watson, Lakshmi N. Yatham, Allan H. Young and Paul R. A. Stokes

https://doi.org/10.1192/bjp.2018.257 Published online by Cambridge University Press, 06 December 2018

The original declaration of interest for this article wrongly implied that Andrea Cipriani (A.C.) had received fees for lecturing from the pharmaceutical companies Lundbeck and Sunovion. This was an accidental duplication of the declaration of interest by Anthony J. Cleare (A.J.C.) which immediately follows it.

Andrea Cipriani has no conflict of interest and nothing to declare. The article has been updated with an explanatory note.
Keywords: Treatment resistant, bipolar disorder, depression, definition, consensus, corrigendum

Diego Hidalgo-Mazzei, Michael Berk, Andrea Cipriani, Anthony J. Cleare, Arianna Di Florio, Daniel Dietch, John R. Geddes, Guy M. Goodwin, Heinz Grunze, Joseph F. Hayes, Ian Jones, Siegfried Kasper, Karine Macritchie, R. Hamish McAllister-Williams, Richard Morriss, Sam Nayrouz, Sofia Pappa, Jair C. Soares, Daniel J. Smith, Trisha Suppes, Peter Talbot, Eduard Vieta, Stuart Watson, Lakshmi N. Yatham, Allan H. Young, Paul R. A. Stokes

\section{Reference}

Hidalgo-Mazzei D, et al. Treatment-resistant and multi-therapy-resistant criteria for bipolar depression: consensus definition. Br J Psychiatry 2019; 214: 27-35. 